\title{
Neural coding of naturalistic motion stimuli
}

\author{
G. D. Lewen, W. Bialek and R. R. de Ruyter van Steveninck \\ NEC Research Institute \\ 4 Independence Way \\ Princeton, New Jersey 08540
}




\begin{abstract}
.
We study a wide field motion sensitive neuron in the visual system of the blowfly Calliphora vicina. By rotating the fly on a stepper motor outside in a wooded area, and along an angular motion trajectory representative of natural flight, we stimulate the fly's visual system with input that approaches the natural situation. The neural response is analyzed in the framework of information theory, using methods that are free from assumptions. We demonstrate that information about the motion trajectory increases as the light level increases over a natural range. This indicates that the fly's brain utilizes the increase in photon flux to extract more information from the photoreceptor array, suggesting that imprecision in neural signals is dominated by photon shot noise in the physical input, rather than by noise generated within the nervous system itself.
\end{abstract}

\title{
1. Introduction
}

One tried and tested way to study sensory information processing by the brain is to stimulate the sense organ of interest with physically appropriate stimuli and to observe the responses of a selected part of the system that lends itself to measurement. Within that framework there are strong incentives, both practical and analytical, to simplify stimuli. After all, short lightflashes or constant tones are easier to generate and to capture mathematically than the everchanging complex world outside the laboratory. Fortunately, sense organs and brains are extremely adaptive, and they apparently function in sensible ways, even in the artificial conditions of typical laboratory experiments. A further reason for using simplified stimuli is that they are presumed to elicit simple responses, facilitating interpretation of the system's inputoutput behaviour in terms of underlying mechanism. Typically, these simple stimuli are repeated a large number of times, the measured outputs are averaged, and this average is defined to be the 'meaningful' component of the response. This is especially helpful in the case of spiking neurons, where we face the embarrasment of the action potential: Because they are an extremely nonlinear feature of the neural response we often do not really know how to interpret sequences of action potentials (Rieke et al , 1997). One way to evade the question and save tractability is to work with derived observables, in particular with smooth functions of time, such as the average firing rate.

Although it certainly is useful to perform experiments with simplified inputs, one also would like to know how those stimuli are processed and encoded that an animal is likely to encounter in nature. We expect animals to be 'designed' for those conditions, and it will be interesting to see to what extent the brain can keep up with the range and strength of these stimuli. In the present work we are concerned primarily with the question of how noisy neural information processing really is. This question cannot be answered satisfactorily if we do not study the problem that the brain is designed to solve, because for us it is hard to distinguish willful neglect on the part of the brain in solving artificial tasks, from noisiness of its components. 
As soon as we try to characterize the behaviour of a sensory system in response to the complex, dynamic, nonrepeated signals presented by the natural world, we lose many of the simplifications mentioned earlier. To meet the challenge we must modify both our experimental designs and our methods for analyzing the responses to these much more complicated inputs. Recent examples of laboratory based approaches to the problem of natural stimulation are studies of bullfrog auditory neurons responding to synthesized frog calls (Rieke et al , 1995), insect olfactory neurons responding to odour plumes (Vickers et al , 2001), cat LGN cells responding to movies (Dan et al, 1996, Stanley et al , 1999), primate visual cortical cells during free viewing of natural images (Gallant et al , 1998, Vinje and Gallant, 2000), auditory neurons in song birds stimulated by song and song-like signals (Theunissen and Doupe, 1998, Theunissen et al , 2000, Sen et al , 2000), the responses in cat auditory cortex to signals with naturalistic statistical properties (Rotman et al , 1999), and motion sensitive cells in the fly (Warzecha and Egelhaaf, 2001, de Ruyter van Steveninck et al , 2001). In each case compromises are struck between well controlled stimuli with understandable statistical properties and the fully natural case.

A more radical approach to natural stimulation was taken by Roeder in the early sixties (see Roeder, 1998). He and his coworkers made recordings from moth auditory neurons in response to the cries of bats flying overhead in the open field. More recently the visual system of Limulus was studied with the animal moving almost free on the sea floor (Passaglia et al, 1997).

Here we study motion sensitive visual neurons in the fly, and - in the spirit of Roeder's work - rather than trying to construct approximations to natural stimuli in the laboratory, we take the experiment into nature. We record the responses of $\mathrm{H} 1$, a wide field direction selective neuron that responds to horizontal motion, while the fly is being rotated along angular velocity trajectories representative for free flying flies. These trajectories indeed are quite wild, with velocities of several thousand degrees per second and direction changes which are complete within ten milliseconds. In analyzing the responses to these stimuli we would like to use methods that do not depend on detailed assumptions about what features of the stimulus are encoded or about what features of the spike sequences carry this coded signal. Recently, information theoretical methods were developed for analysing neural responses to repeated sequences of otherwise arbitrarily complex stimuli (de Ruyter van Steveninck et al , 1997, Strong et al , 1998). In our experiments we repeat the same motion trace, lasting several seconds, and this provides us with the raw data for computing the relevant information measures, as explained in section 2.3. We emphasize that although we repeat the stimulus many times to estimate the relevant probability distributions of responses, the measures we derive from these distributions characterize the information coded by a single example of the neural response. 


\section{Methods}

\subsection{Stimulus design considerations}

The giant motion sensitive interneurons in the fly's lobula plate are sensitive primarily to such rigid rotational motions of the fly as occur during flight (Hausen, 1982, Krapp et $a l, 1997)$, and these cells typically have very large visual fields. It is this wide field rigid rotation that we want to reproduce as we construct a naturalistic stimulus. But what pattern of rotational velocities should we use? As a benchmark we will present data from an experiment where the fly was rotated at velocities that remained constant for one second each. We would, however, also like to present the fly with stimuli that are more representative for natural flight. Free flight trajectories were recorded in the classic work of Land and Collett (1974), who studied chasing behaviour in Fannia canicularis and found body turning speeds of several thousand degrees per second. A recent study (van Hateren and Schilstra, 1999) reports flight measurements from Calliphora at high temporal and spatial resolution. In these experiments flies made of order 10 turns per second, and turning speeds of the head reached values of over $3000^{\circ} / \mathrm{s}$. In general, high angular velocities pose problems for visual stimulus displays, because even at relatively high frame rates they may give rise to ghost images. In our laboratory we use a Tektronix 608 display monitor with a $500 \mathrm{~Hz}$ frame rate,. Then $3000^{\circ} / \mathrm{s}$ corresponds to jumps from frame to frame of $6^{\circ}$, four times larger than the spacing between photoreceptors. Although the frame rate used here is well above the photoreceptor flicker fusion frequency (de Ruyter van Steveninck and Laughlin, 1996) the presence of ghost images may have consequences for the encoding of motion signals. Further, the light intensity of the typical displays used in the laboratory is much lower than

outside. As an example, the Tektronix 608 induces of order $5 \cdot 10^{4}$ photoconversions/s in fly photoreceptors at its maximum brightness. The brightness outside can easily be a factor of a hundred higher (Land, 1981), although the photoreceptor pupil mechanism will limit the maximum photon flux to about $10^{6}$ photoconversions/s (Howard et al, 1987). Finally, the field of view of H1 is very large, covering essentially the field of one eye (Krapp and Hengstenberg, 1997), which is about $6.85 \mathrm{sr}$ or $55 \%$ of the full $4 \pi$ sr in female Calliphora vicina (estimates based on Beersma et al , 1977. See also Fig. 1). In practice with a display monitor it is hard to stimulate the fly with coherent motion over such a large area and in most of our laboratory experiments we stimulate less than about $20 \%$ of the full visual field of $\mathrm{H} 1$.

\subsection{Stimulus apparatus}

All the factors mentioned above suggest an experimental design in which the visual world can be made to move more or less continuously relative to the fly, and this is easiest to accomplish by moving the fly relative to the world as occurs during free flight. We therefore constructed a light and compact assembly consisting of a fly holder, electrode manipulator, and preamplifier that can be mounted on a stepper motor, as shown in 
figure 1. This setup is rigid enough to allow high speed rotations around the vertical axis while extracellular recordings are made from the $\mathrm{H} 1$ cell. Because it is powered by batteries the setup can be taken outside, so that the fly's visual system is stimulated with natural visual scenes. The mounting and recording stage inevitably covers some area in the fly's visual field. During the experiment this rotates along with the fly, and so does not contribute to motion in the fly's visual field. By tracing the contours of the setup as seen from the fly, we estimate the shape and size of this overlap, as depicted in figure 1. The setup was designed to minimize the overlap in the visual field of the left eye. In the experiments presented here, recordings were therefore made from contralateral H1, on the right side of the head. The setup occludes only $1.52 \mathrm{sr}$, or $22 \%$, of the visual field of the left eye, most of it ventral-caudal, as indicated by the heavy mesh in the right panel of figure 1.

The stepper motor (Berger-Lahr RDM 564/50) was driven by a Divistep 331.1 controller in microstep mode, that is, at $10^{4}$ steps per revolution, corresponding to a smallest step size of $0.036^{\circ}$ or roughly $1 / 30$ th of an interommatidial angle. The Divistep controller in turn was driven by pulses from a custom designed interface that produced pulse trains by reading pulse frequency values from the parallel port of a laptop computer. Pulse frequency values were refreshed every $2 \mathrm{~ms}$.

To generate naturalistic motion stimuli we used published trajectories of chasing Fannia from Land and Collett (1974), interpolated smoothly between their 20 ms sample points. For technical reasons we had to limit the accelerations of the setup, and we chose therefore to rotate the fly at half the rotational velocities derived from the Land and Collett data. This may be reasonable as Calliphora is a larger fly than Fannia, and is likely to make slower turns. The constant velocity data presented in figure 2 were taken with rotation speeds ranging from about $0.28^{\circ} / \mathrm{s}$ to $4500^{\circ} / \mathrm{s}$. To avoid extreme accelerations during high velocity presentations the pulse program for the stepper motor delivered smooth $100 \mathrm{~ms}$ pulse frequency ramps to switch between velocities. For velocities below $18^{\circ} / \mathrm{s}$ pulses were sent to the controller at intervals longer than $2 \mathrm{~ms}$. At the lowest constant velocity used in our experiments, $0.28^{\circ} / \mathrm{s}$, pulses were delivered at $128 \mathrm{~ms}$ intervals. The step size was small enough so that a modulation of the PSTH was undetectable in the experiment.

The experiment of figure 2 compares data from the outdoor setup to data taken inside with the fly observing a Tektronix 608 CRT. The stimulus displayed on this monitor consisted of 190 vertical lines, with intensities derived from a one-dimensional scan of the scene viewed by the fly in the outdoor experiment. The moving scene was generated by a digital signal processor, and written at a $500 \mathrm{~Hz}$ frame rate. As mentioned above, this gives rise to ghosting at high image speeds when the pattern makes large jumps from frame to frame. The DSP produced the coarse part of motion essentially by stepping through lines in a buffer memory. On top of this, fine displacements were produced by moving the entire image by fractions of a linewidth at each frame. The resulting motion was smooth and not limited to integer steps. The fly was positioned so that the screen subtended a rectangular area of $67^{\circ}$ horizontal by $55^{\circ}$ vertical, with 
the left eye facing the CRT and rightmost vertical edge of the CRT approximately in the sagittal plane of the fly's head.

\subsection{Information theoretic analysis of neural firing patterns}

We describe briefly a technique for quantifying information transmission by spike trains (de Ruyter van Steveninck et al , 1997, Strong et al , 1998, de Ruyter van Steveninck et al , 2001). We consider segments of the spike train with length $T$ divided in a number of bins of width $\Delta t$, where $\Delta t$ ranges from one millisecond up to $\Delta t=T$. Each such bin may hold a number of spikes, but within a bin no distinction is made on where the spikes appear. However, two windows of length $T$ that have different combinations of filled bins are counted as different firing patterns. Also, two windows in which the same bins are filled but with different count values, are distinguished. We refer to such firing patterns as words, $W_{T, \Delta t}$. From an experiment in which we repeat a reasonably long naturalistic stimulus a number of times, $N_{r}$ (here $N_{r}=200$ repetitions of a $T_{r}=5$ seconds long sequence) we get a large number of these words, $W_{T, \Delta t}(t)$, with $t$ the time since the start of the experiment. Here we discretize $t$ in $1 \mathrm{~ms}$ bins, giving us 5000 words per repetition period, and $10^{6}$ words in the entire experiment. From this set of words we set up word probability distributions, from which we calculate total and noise entropies, and their difference, according to Shannon's definitions:

(i) The total entropy, $S_{t o t}(T, \Delta t)$. From the list of words $W_{T, \Delta t}(t)$, for all $t(0 \leq t \leq$ $\left.N_{r} \cdot T_{r}\right)$, we directly get a distribution, $P\left(W_{T, \Delta t}\right)$ describing the probability of finding a word anywhere in the entire experiment. The total entropy is now:

$$
S_{t o t}(T, \Delta t)=-\sum_{W} P\left(W_{T, \Delta t}\right) \cdot \log _{2}\left[P\left(W_{T, \Delta t}\right)\right]
$$

This entropy measures the richness of the 'vocabulary' used by H1 under these experimental conditions, hence the time of occurrence of the pattern within the experiment is irrelevant.

(ii) The average noise entropy, $\bar{S}_{\text {noise }}(T, \Delta t)$. If the neuron responded perfectly reproducibly to repeated stimuli, then the information conveyed by the spike train would equal the total entropy defined above. There is noise, however, and this leads to variations in the responses, as can be seen directly from the rasters in Fig. 3. $\bar{S}_{n o i s e}(T, \Delta t)$ gives us an estimate of how variable the response to identical stimuli is. We first accumulate, for each instant $t_{r}$ in the stimulus sequence, the distribution of all those firing patterns $P\left(W_{T, \Delta t} \mid t_{r}\right)$, taken across all trials, that begin at $t_{r}$ (note that $0 \leq t_{r} \leq T_{r}$ ). The entropy of this distribution measures the (ir)reproducibility of the response at each instant $t_{r}$ :

$$
S_{n o i s e}\left(T, \Delta t, t_{r}\right)=-\sum_{W} P\left(W_{T, \Delta t} \mid t_{r}\right) \cdot \log _{2}\left[P\left(W_{T, \Delta t} \mid t_{r}\right)\right]
$$

Calculating this for each point in time and averaging all these values we obtain the average noise entropy:

$$
\bar{S}_{\text {noise }}(T, \Delta t)=\frac{1}{T_{r}} \int_{0}^{T_{r}} S_{\text {noise }}\left(T, \Delta t, t_{r}\right) d t_{r}
$$


(iii) The information conveyed by words at the given length $T$ and resolution $\Delta t$ is the difference of these two entropies:

$$
I(T, \Delta t)=S_{t o t}(T, \Delta t)-\bar{S}_{n o i s e}(T, \Delta t) .
$$

The coding efficiency of the spike train is the fraction of the total entropy that is utilized to convey information:

$$
\eta(T, \Delta t)=\frac{I(T, \Delta t)}{S_{t o t}(T, \Delta t)} .
$$

Small values of $\eta(T, \Delta t)$ indicate a loose coupling between stimulus and spike train, whereas values close to 1 imply that there is little noise entropy, so that most of the structure of the spike train is meaningful, and carries a message. Here we will not be interested in the decoding question, that is in what that message is, but only in how much information is conveyed about the stimulus. We will then compare these values in different conditions.

It should be stressed that the information values we derive by these methods are not strictly about velocity. They are potentially about anything in the stimulus that is repetitive with period $T_{r}$. It is our job as experimenters to construct inputs that we think will stimulate the neuron well, and for H1, naturalistic wide field motion seems to be a good choice. But that does not necessarily mean that that is the best choice. Further, the motion pattern is dynamic, and any noiseless time invariant operation on this signal will produce a result that has the same repeat period as the original. Our information measures do not distinguish these cases; specifically, our discussion is unaffected by the question of whether $\mathrm{H} 1$ encodes velocity, acceleration, or some nonlinear function of these variables. Questions of decoding are highly interesting, but at the same time difficult to tackle for stimuli of the type studied here, and we will leave them aside in this paper.

It is interesting to try and estimate $I(T, \Delta t)$ as we let $T$ become very long, and $\Delta t$ very short, as this limit is the average rate of information transmission. Because calculating this limit requires very large data sets, we focus here on the information transmitted in constant time windows, $T=30 \mathrm{~ms}$, as a function of $\Delta t$. We choose $T=30 \mathrm{~ms}$ because that amounts to the delay time with which a chasing fly follows turns of a leading fly during a chase (Land and Collett, 1974); the end result, that is the dependence of information transmission on $\Delta t$, was found not to depend critically on the choice of $T$.

To quantify noise entropy, the method described above requires that a stimulus waveform be repeated. Although it is possible in principle to quantify information transmission based only on one repetition, using many repetitions is easier in practice. In a sense this mode of stimulation is still removed from the realistic situation in which stimuli are not repeated at all. Indeed, in our experiments there are hints that the fly adapts to the stimulus somewhat over the first few presentations of the 5 second long stimulus. The effects of adaptation to dynamic stimuli are certainly interesting (Brenner et al , 2000, Fairhall et al , 2001), but in the data we present here we skip the first 
few presentations, and only analyze that part of the experiment in which the fly seems fully adapted to the ongoing dynamic stimulus. Inspection of the rasters in that phase shows no obvious trends, so that the fly seems to be close to stationary conditions. In this regard our information measures are lower bounds, as deviations from stationarity will increase our estimate of the noise entropy, lowering information estimates.

\section{Results}

\subsection{Operating range for naturalistic motion stimuli}

In order to be sure that $\mathrm{H} 1$ receives no dominant motion related signals from other modalities than vision we rotated the fly either in darkness or under a cover that turned along with the fly. This did not produce discernible motion responses in H1. Strictly speaking that does not exclude possible modulatory mechanosensory input, which could be investigated in principle by presenting conflicting visual and mechanosensory stimuli. The possibility seems remote, however, and even if true it would not invalidate our conclusions about the dependence of H1's information transmission on parameters of the visual stimulus.

As a first comparison between laboratory and natural conditions we present data from an experiment in which $\mathrm{H} 1$ was excited by one second long episodes of motion at constant velocity. These were presented at a range of velocities from about $0.28^{\circ} / \mathrm{s}$ to $4500^{\circ} / \mathrm{s}$. Outdoors the fly was placed in a wooded environment and rotated on the stepper motor. In the laboratory the same fly watched a vertical bar pattern derived from a one dimensional scan of the natural environment in which the outdoor experiment was done. This pattern was displayed on a standard Tektronix 608 monitor, with a rectangular stimulated visual area of $67^{\circ}$ horizontal by $55^{\circ}$ vertical. The pattern moved at the same settings of angular velocity as were used outdoors, but the indoor and outdoor stimuli differed both in average light level and in stimulated area.

Figure 2 shows the average firing rates obtained from the last half second of each velocity presentation. At low velocities, up to about $20^{\circ} / \mathrm{s}$, the spike rates for both conditions are not very different, despite the large change in total motion signal present in the photoreceptor array. Apparently the fly adapts these differences away (see Brenner et $a l, 2000)$. In both experiments the rate depends roughly logarithmically on velocity over an appreciable range and this is partly a result of adaptation as well (de Ruyter van Steveninck et al , 1986). In the laboratory experiment the motion response peaks at about $100^{\circ} \mathrm{s}$, whereas in natural conditions the fly encodes velocities monotonically for

an extra order of magnitude, its response peaking in the neighbourhood of $1000^{\circ} / \mathrm{s}$. This brings H1's encoding of motion under natural conditions in the range of behaviourally relevant velocities. A lack of sensitivity to high speeds has been claimed both to be an essential result of the computational strategy used by the fly, and to be advantageous in optomotor course control (Warzecha and Egelhaaf, 1998). These conclusions do not pertain to the conditions in the outdoor experiment, where $\mathrm{H} 1$ responds robustly and 
reliably to angular velocities of well over $1000^{\circ} / \mathrm{s}$.

\subsection{Motion detection throughout the day}

Figure 3 shows spike train rasters generated by $\mathrm{H} 1$ in three outdoor experiments, focusing on a short segment that illustrates some qualitative points. Trace (a) shows the velocity waveform, which was the same in all three cases. The experiments were performed at noon (b), half an hour before sunset (c), and about half an hour after sunset (d). Rough estimates of the photon flux in a blowfly photoreceptor looking at zenith are shown beside the panels. In all experiments the fly saw the same scene, with a spatial distribution of intensities ranging from about $5 \%$ to $100 \%$ of the zenith value.

The figure reveals that some aspects of the response are quite reproducible, and further that particular events in the stimulus can be associated reliably with small numbers of spikes. More dramatically, the timing precision of the spike trains gradually decreases going from the noon experiment to the one after sunset. Higher photon rates imply a more reliable physical input to the visual system. The figure therefore strongly suggests that the fly's visual system utilizes this increased input reliability to compute and encode motion more accurately when the light intensity increases. This statement is ecologically relevant, as the conditions of the experiment correspond to naturally occurring light levels and approximately to the naturally stimulated visual area. To get a feeling for the spike timing precision in the three conditions we can simply look at the distribution of timing of the first spike generated after a fixed criterion time (for which we choose $t=0.28 \mathrm{~s}$ in (b) and (c) and $\mathrm{t}=0.30 \mathrm{~s}$ in (d)). The jitter in the spike timing across different trials has a standard deviation of $0.95 \mathrm{~ms}$ in (b), $1.4 \mathrm{~ms}$ in (c), and 5.8 $\mathrm{ms}$ in (d). The relative timing of spikes can be even more accurate: the interval from the first to the second spike fired after the criterion time is $2.3 \pm 0.23 \mathrm{~ms}$ in (b), $5.0 \pm 0.6$ $\mathrm{ms}$ in (c), and $16 \pm 2.4 \mathrm{~ms}$ in (d). Compared to the rapid onsets and offsets of the spike activity at the higher photon fluxes, the stimulus varies rather smoothly, which means that the time definition of spikes with respect to the stimulus can be much better than might be suggested by the stimulus bandwidth. An example can be seen in the rather smooth hump in the velocity waveform at about $\mathrm{t}=0.43 \mathrm{~s}$, which induces on most trials a well defined response consisting of a sharply defined pair of spikes.

We quantify these impressions using the information theoretic approach described briefly in section 2.3. The result of this analysis is shown in Fig. 4a-d, for the three different experiments discussed above. Figure $4 \mathrm{c}$ clearly shows that the information in a 30 ms window increases both when the light intensity goes up, and when the spikes are timed with higher accuracy. The increase in information with increasing spike timing precision is most dramatic for the highest light levels, indicating that coding by fine spike timing becomes more prominent the better the input signal to noise ratio. A comparison of figures 4a (total entropy) and figure $4 \mathrm{~b}$ (noise entropy) reveals that the increase in information content with increasing light levels is primarily due to an increase in total entropy: The neuron's vocabulary increases in size as its input becomes better 
defined. Figure $4 \mathrm{~d}$ shows that at the two highest light intensities the coding efficiency is of order 0.5 at time resolution $\Delta t=1 \mathrm{~ms}$, increasing slightly for larger values of $\Delta t$. In the darkest condition the efficiency decreases markedly for all values of time resolution.

The right column of figure 4 compares experiments in which we took data both outdoors and in the laboratory. These data are from another fly, but the conditions of the outdoor experiment were similar to those for the first fly at the highest light level. After the outdoor experiment the fly was taken inside the laboratory, and the same velocity stimulus as the one used outside was repeated inside. In the laboratory, as before, the visual stimulus was presented on a Tektronix 608 CRT. Photoreceptors facing the CRT received about $5 \cdot 10^{4}$ photons per second at maximum intensity, a value in between the light intensities seen by the first fly in the experiments just before and just after sunset (grey and black symbols in figure 4a-d). Two experiments were done indoor, one in which the picture on the monitor consisted of vertical bars with a contrast pattern measured in a horizontal scan of the outdoor scene (filled triangles), the other a high contrast square wave pattern with contrast $=1$, and spatial wavelength $=12.5^{\circ}$ (filled squares). From figure $4 \mathrm{~g}$ we see that the information transmitted by $\mathrm{H} 1$ is much lower in the laboratory experiments than in the outdoor experiment, due to the smaller stimulated area and the lower light level. Figure 4e shows that the decrease in information, as before, is mainly due to a lowering of the total entropy. The noise entropy also decreases (figure 4f), but not enough to compensate. Somewhat surprisingly, the experiment with the high contrast pattern indoors leads to a slightly higher coding efficiency than even the outdoor experiment.

\section{Discussion}

Outdoor illumination can easily be a hundred times brighter than anything displayed on common laboratory equipment, and in the outdoor experiment stimuli extend over a large fraction of the fly's full visual field rather than being confined to a small flat monitor. Both effects are relevant for our experiments, as the higher brightness leads to higher photoreceptor signal to noise ratios (de Ruyter van Steveninck and Laughlin, 1996), and as H1's receptive field covers almost a hemisphere (Krapp and Hengstenberg, 1997). In moving from laboratory to outdoor conditions, both effects increase the signal to noise ratio of the input available for computation of rigid wide field motion from the photoreceptor array. The question then is whether the fly's brain uses this improvement in input signal quality to produce more accurate estimates of visual motion, and/or increase its operating range of motion detection. Figure 2 shows that the range of velocities that are encoded increases markedly when the visual input becomes more reliable.

If the accuracy of information processing is limited by noise sources within the nervous system, we should observe a plateau, that is, information transmission should saturate at some defined level of input signal quality. There is some arbitrariness in the choice of the level of input signal quality, however: In principle we can surpass any degree 
of accuracy of the physical input signal by simply increasing the light intensity, and at some point the internal randomness of the brain's components must become the limiting factor in information processing. However, statements about the magnitude of internal versus external noise in sensory information processing are primarily meaningful in the context of reasonable, physiological levels of input signal quality. Those stimuli that the animal encounters naturally, taken at the high end of their dynamic range, would meet this criterion. For the case considered here the dynamic range refers to light intensity, size of stimulated visual field, and dynamics of motion. The data we recorded outdoors show no sign of saturation in information transmission when the input signal quality increases. On the contrary, if we compare the rasters of figure $3 \mathrm{~b}$ and $3 \mathrm{c}$, we see that there is a marked improvement in the timing of spikes, even over the highest decade of light intensity $\left(2 \times 10^{5}\right.$ to $3 \times 10^{6}$ photons/s at zenith per photoreceptor $)$. This improvement translates into a significant gain in information transmission, especially at fine time resolution, as shown in figure 4c. Thus, in computing motion from the array of photoreceptors, the fly's brain does not suffer noticeably from information bottlenecks imposed by internal noise, under ecologically relevant conditions.

In our outdoor experiments, the information content of the spike train varies primarily as a result of a varying total entropy (figure 4a). The noise entropy (figure 4b) appears to be almost constant as a function of light level. One can distinguish two different ways to increase information transmission through a channel. The first is to encode the same messages more accurately, the second to increase the variety of messages, keeping the accuracy of each individual message the same. The first scheme implies constant total entropy and decreasing noise entropy, the second an increase in total entropy at constant noise entropy. Our data suggest that as the visual input becomes more reliable, the fly chooses to increase the vocabulary of $\mathrm{H} 1$ to encode a wider variety of features of the motion stimulus, keeping precision roughly constant.

Acknowledgments We thank Naama Brenner, Steve Strong and Roland Koberle for many pleasant and enlightening discussions.

\section{References}

Beersma, D.G.M., Stavenga, D.G., and Kuiper, J.W. Retinal lattice, visual field and binocularities in flies. Dependence on species and sex. J. Comp. Physiol. 119, 207-220 (1977).

Brenner, N., Bialek, W., and de Ruyter van Steveninck, R. Adaptive rescaling maximizes information transmission, Neuron 26, 695-702 (2000).

Dan, Y., Atick, J. J., and Reid, R. C. Efficient coding of natural scenes in the lateral geniculate nucleus: Experimental test of a computational theory, J. Neurosci. 16, 3351-3362 (1996).

Fairhall, A.L., Lewen, G.D., Bialek, W., de Ruyter van Steveninck, R.R. Multiple timescales of adaptation in a neural code. To be published in Advances In Neural Information Processing Systems $14,2001$.

Gallant, J. L., Conner, C. E., and van Essen, D C. Neural activity in areas V1, V2 and V4 during free viewing of natural scenes compared to controlled viewing NeuroReport 9, 1673-1678 (1998). 
van Hateren, J. H., and Schilstra, C. Blowfly flight and optic flow II. Head movements during flight, J. Exp. Biol. 202, 1491-1500 (1999).

Hausen, K. Motion sensitive interneurons in the optomotor system of the fly. II. The horizontal cells: Receptive field organization and response characteristics. Biol. Cybern. 46, 67-79 (1982).

Howard, J., Blakeslee, B., Laughlin, S.B. The intracellular pupil mechanism and the maintenance of photoreceptor signal to noise ratios in the blowfly Lucilia cuprina. Proc. R. Soc. Lond. B. 231, 415-435 (1987).

Krapp, H.G., and Hengstenberg, R. A fast stimulus procedure to determine local receptive field properties of motion-sensitive visual interneurons. Vision Research 37, 225-234 (1997).

Land, M. F. Optics and Vision in Invertebrates. In: Autrum, H. (ed) Handbook of Sensory Physiology. Springer, Berlin, Heidelberg, New York (1981), pp. 472-592.

Land, M. F., and Collett, T. S. Chasing behavior of houseflies (Fannia canicularis). A description and analysis, J Comp Physiol 89, 331-357 (1974).

Passaglia C., Dodge F., Herzog E., Jackson S., Barlow R. (1997): Deciphering a neural code for vision. Proc Natl Acad Sci USA 94, 12649-12654.

Rieke, F., Bodnar, D. and Bialek, W. Naturalistic stimuli increase the rate and efficiency of information transmission by primary auditory neurons. Proc. R. Soc. Lond. B 262, 259-265.

Rieke, F., Warland, D., de Ruyter van Steveninck, R. R., and Bialek, W. Spikes: Exploring the neural code (MIT Press, Cambridge, 1997).

Roeder, K.D. (1998): Nerve cells and insect behavior. Harvard University Press, Cambridge, MA.

Rotman, Y., Bar Yosef, O., and Nelken, I. Responses of auditory-cortex neurons to structural features of natural sounds, Nature 397, 154-157 (1999).

de Ruyter van Steveninck, R.R., Zaagman, W.H., Mastebroek, H.A.K. Adaptation of transient responses of a movement sensitive neuron in the visual system of the blowfly Calliphora erythrocephala. Biol. Cybern. 54, 223-236 (1986).

de Ruyter van Steveninck, R.R., Laughlin, S.B.: Light adaptation and reliability in blowfly photoreceptors. Int. J. Neural. Syst. 7, 437-444. (1996).

de Ruyter van Steveninck, R. R., Lewen, G. D., Strong, S. P., Koberle, R., and Bialek, W. Reproducibility and variability in neural spike trains. Science 275, 1805-1808 (1997).

de Ruyter van Steveninck, R., Borst, A., Bialek, W. real-time encoding of motion: Answerable questions and questionable answers from the fly's visual system. In: J.M. Zanker and J. Zeil (eds.) Motion vision. Computational and ecological constraints. Springer, Berlin, Heidelberg, New York (2001), pp. 279-306.

Sen, K., Wright, B. D., Bialek, W., and Doupe, A. J. Discovering features of natural stimuli relevant to forebrain auditory neurons. Soc. Neurosci. Abstract 551.2 (2000).

Stanley, G. B., Li, F. F., and Dan, Y. Reconstruction of natural scenes from ensemble responses in the lateral geniculate nucleus, J. Neurosci. 19, 8036-8042 (1999).

Strong, S. P., Koberle, R., de Ruyter van Steveninck, R. R., and Bialek, W. Entropy and information in neural spike trains. Phys Rev Lett 80, 197-200 (1998).

Theunissen, F. E., and Doupe, A. J. Temporal and spectral sensitivity of complex auditory neurons in the nucleus HVc of male zebra finches, J. Neurosci. 18, 3786-3802 (1998).

Theunissen, F. E., Sen, K., and Doupe, A. J. Spectral-temporal receptive fields of nonlinear auditory neurons obtained using natural sounds, J. Neurosci. 20, 2315-2331 (2000).

Vickers, N.J., Christensen, T.A., Baker, T. , and Hildebrand, J.G. Odour-plume dynamics influence the brain's olfactory code. Nature 410, 466-470 (2001).

Vinje, W. E., and Gallant, J. L. Sparse coding and decorrelation in primary visual cortex during natural vision, Science 287, 1273-1276 (2000).

Warzecha, A.-K., and Egelhaaf, M. On the performance of biological movement detectors and ideal velocity sensors in the context of optomotor course stabilization, Vis. Neurosci. 15, 113-122 (1998).

Warzecha, A.-K., and Egelhaaf, M. Neural encoding of visual motion in real-time. In: J.M. Zanker and J. Zeil (eds.) Motion vision. Computational and ecological constraints. Springer, Berlin, Heidelberg, 
New York (2001), pp. 239-278. 


\section{Figure legends}

Figure 1. Left: Setup used in the outdoor experiments. The fly is in a plastic tube, head protruding, and immobilized with wax. A small feeding table is made, from which the fly can drink sugarwater. The part of the setup shown here rotates around the axis indicated at the bottom, by means of a stepper motor. A silver reference wire makes electrical contact with the body fluid, while a tungsten microelectrode records action potentials extracellularly from H1, a wide-field motion sensitive neuron in the fly's lobula plate. The electrode signals are preamplified by a Burr-Brown INA111 integrated instrumentation amplifier, the output of which is fed through a slip ring system to a second stage amplifier and filter and digitized by a National Instruments PCMCIA data acquisition card in a laptop computer. The part of the setup visible in the figure is mounted on a stepper motor which is driven by computer-controlled laboratory built electronics. Right: Occlusion in the left visual field of the fly. The dot in centre represents the position of the fly. The animal is looking in the direction of the arrow and has the same orientation as the fly in the setup at left. The thin mesh bordered by the heavy line represents the excluded part of the visual field of the left eye for a free flying fly (based on Beersma et al. 1977). The heavy mesh represents the overlap of the left eye's natural visual field with those parts of the setup that rotate along with the fly, and therefore do not contribute to a motion signal. The total visual field of the left eye is $6.85 \mathrm{sr}$, or $0.55 \cdot 4 \pi$. The overlap depicted by the heavy mesh subtends about $1.52 \mathrm{sr}$, or $22 \%$ of the visual field of the left eye.

Figure 2. A comparison of responses to constant velocity in a typical laboratory experiment (closed squares), and in an outdoor setting where the fly is rotating (open circles). Average firing rates were computed over the last 0.5 seconds of a 1 second constant velocity presentation.

Figure 3. Responses of the $\mathrm{H} 1$ neuron to the same motion trace recorded outside at different times of the day. (a) Short segment of the motion trace executed by the stepper motor with the fly. The full segment of motion lasted 5 seconds, and was derived from video recordings of natural fly flight during a chase (see Methods) (b) 50 Spike rasters in response to the motion trace in (a), taken at noon. (c) As (b), but recorded about half an hour before sunset. (d) As (b), but recorded about half an hour after sunset. 
Figure 4. Lefthand column: Information theoretic quantities for the three outdoor experiments whose rasters are shown in figure 3. The symbol shadings refer to the different conditions of illumination in the experiments. All figures refer to a $30 \mathrm{~ms}$ measurement window in which neural firing patterns are defined at time resolutions, $\Delta t$, of $1,2,3,5,10,15$, and $30 \mathrm{~ms}$, as given by the abscissae. (a): Total entropy of spike firing patterns. (b): Average noise entropy. (c): Average information transmitted by firing patterns. (d): Coding efficiency, defined as the transmitted information divided by the total entropy.

Righthand column: The same quantities as plotted in the lefthand column, but now for an experiment outdoors (open symbols), and two experiments in the laboratory (closed symbols, see text for further description of conditions). Squares are for a moving square wave pattern of high contrast $(\mathrm{C}=1)$, and spatial wavelength $12.5^{\circ}$; triangles are for a moving sample of the natural scene at the location where the outdoor experiments were done. Both these stimulus patterns were generated on the cathode ray tube in the laboratory. 
This figure "fig1.jpg" is available in "jpg" format from: http://arxiv.org/ps/physics/0103088v1 


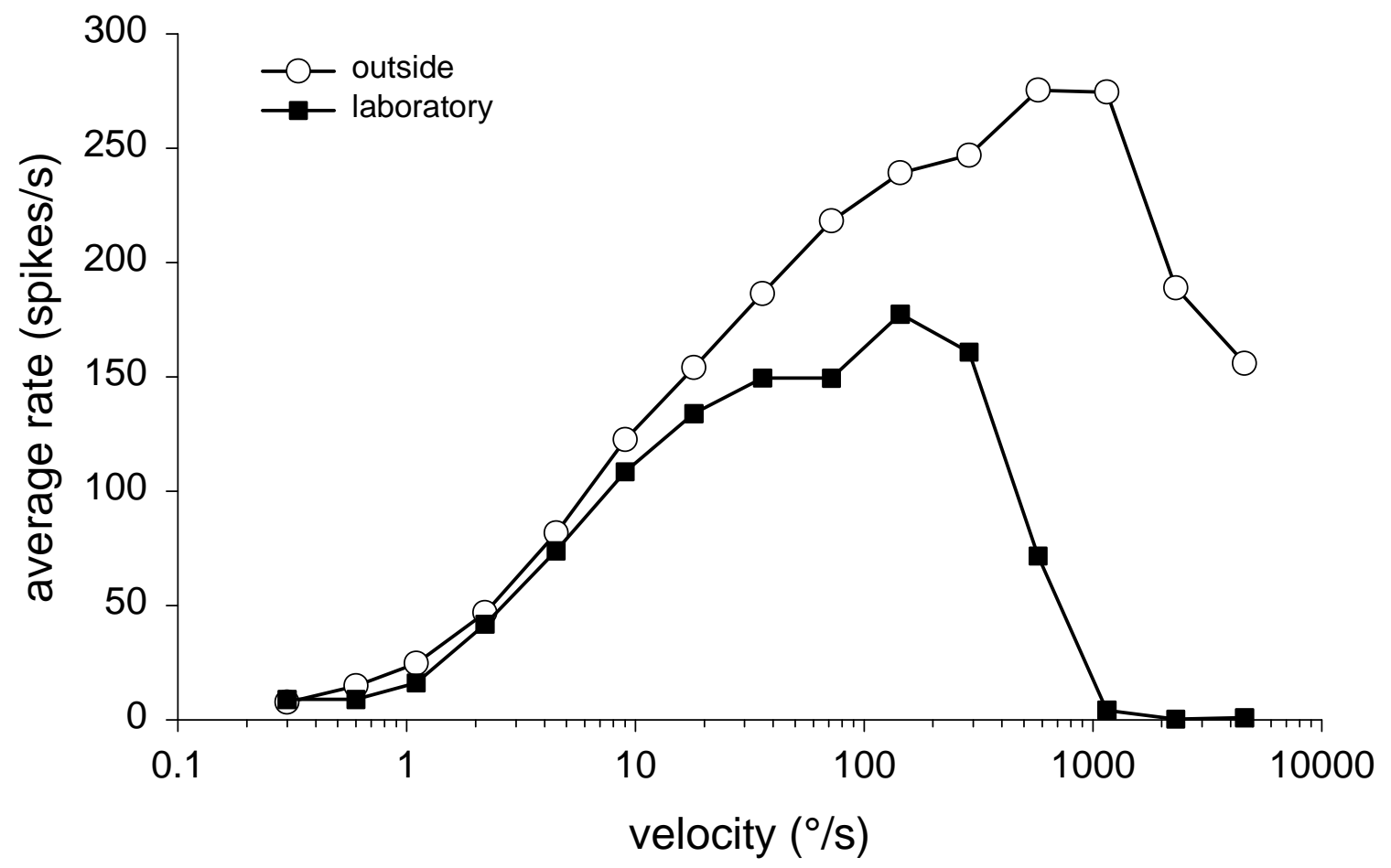


This figure "fig3.jpg" is available in "jpg" format from: http://arxiv.org/ps/physics/0103088v1 
zenith photon flux

(photons/s per photoreceptor)

- $3 \times 10^{2}$

$-2 \times 10^{5}$

a

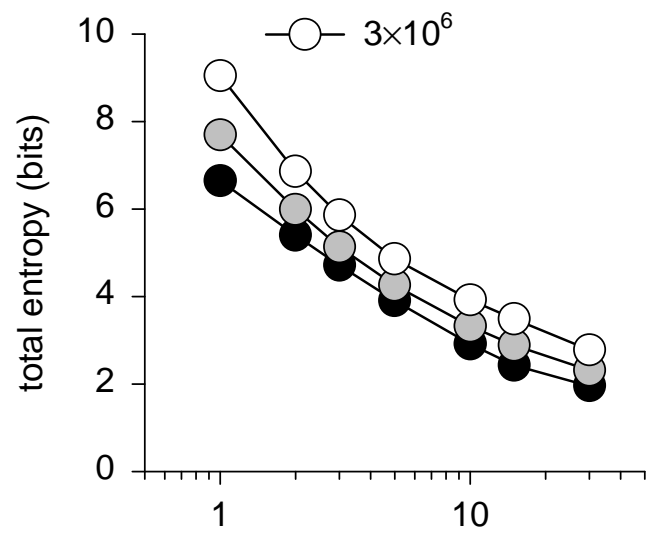

b

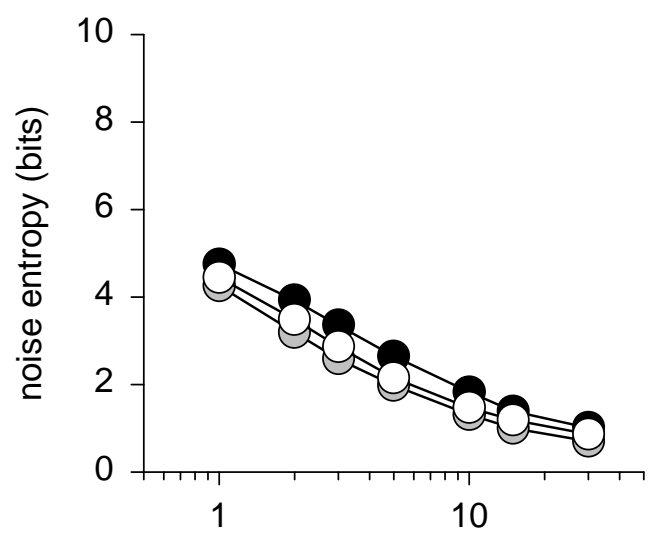

C

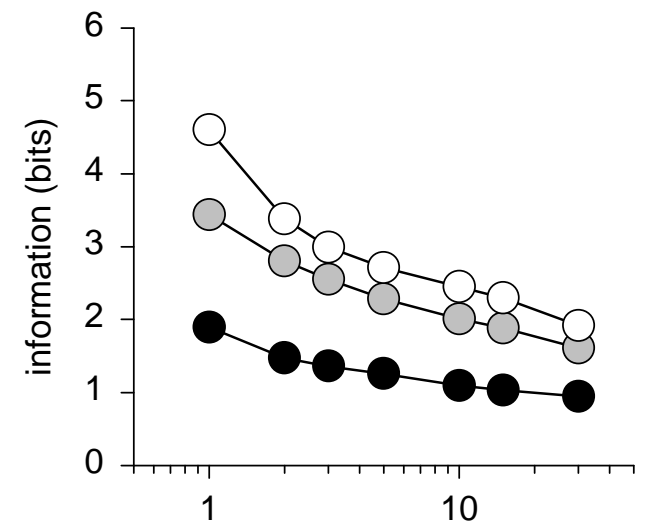

d

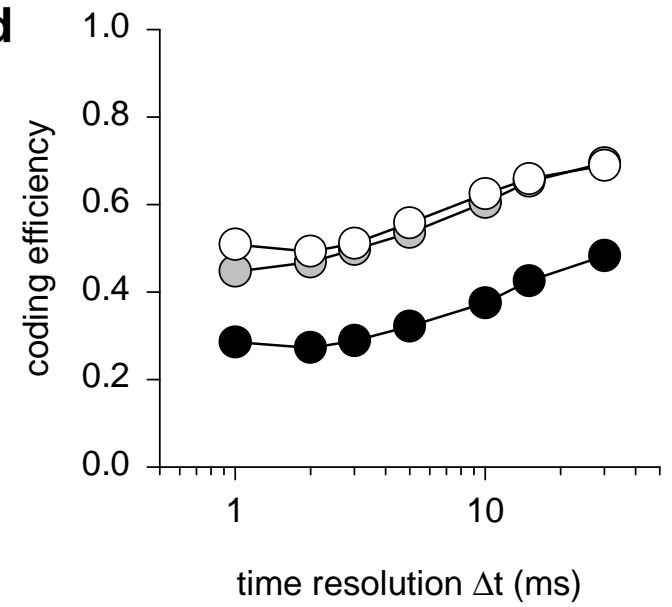

location and stimulus pattern

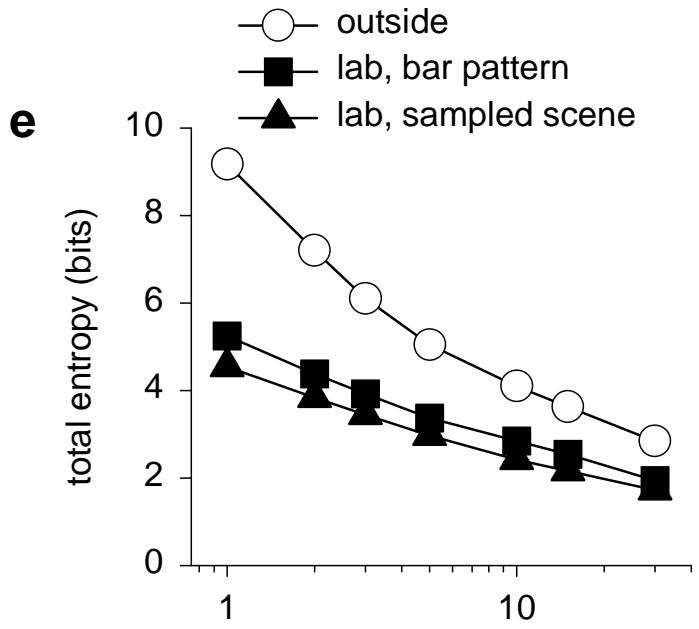

f

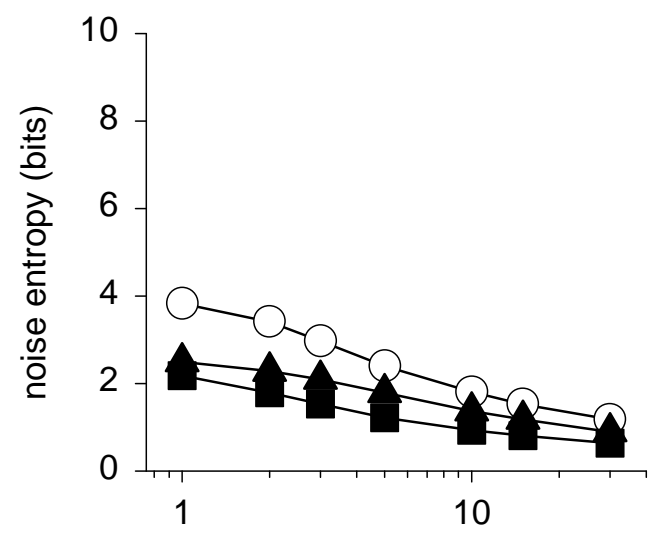

g

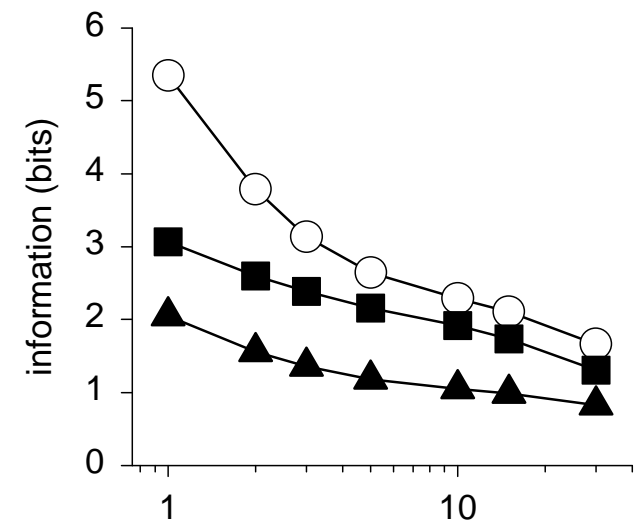

h

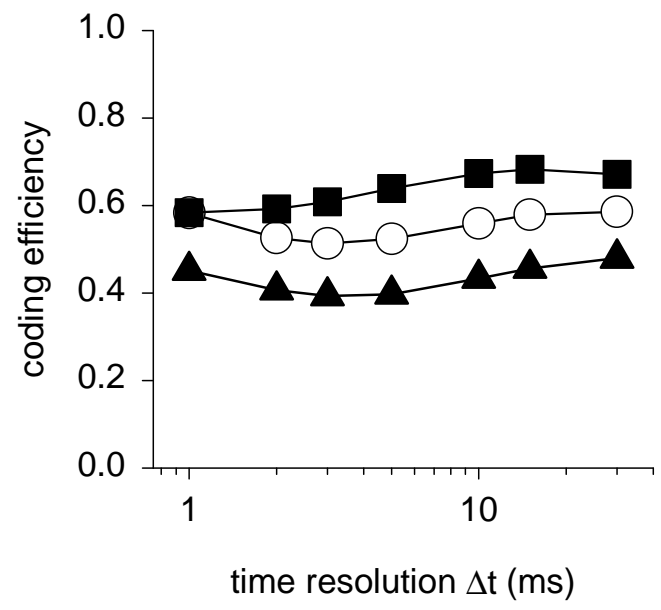

